\title{
BMJ Open Transformation of identity in substance use as a pathway to recovery and the potential of treatment for hepatitis C: a systematic review protocol
}

\author{
Sarah R Donaldson (i) , ${ }^{1,2}$ Andrew Radley (1) , , John F Dillon ${ }^{1,3}$
}

To cite: Donaldson SR, Radley A, Dillon JF. Transformation of identity in substance use as a pathway to recovery and the potential of treatment for hepatitis C: a systematic review protocol. BMJ Open 2022;12:e049713. doi:10.1136/ bmjopen-2021-049713

- Prepublication history and additional supplemental material for this paper are available online. To view these files, please visit the journal online (http://dx.doi.org/10.1136/ bmjopen-2021-049713).

Received 02 February 2021 Accepted 06 January 2022

Check for updates

(C) Author(s) (or their employer(s)) 2022. Re-use permitted under CC BY-NC. No commercial re-use. See rights and permissions. Published by BMJ.

${ }^{1}$ School of Medicine, University of Dundee, Dundee, UK ${ }^{2}$ Directorate of Public Health, NHS Tayside, Dundee, UK

${ }^{3}$ Department of

Gastroenterology, NHS Tayside, Dundee, UK

Correspondence to

Sarah R Donaldson;

Sarah.Donaldson@nhs.scot

\section{ABSTRACT}

Introduction Hepatitis $\mathrm{C}$ virus (HCV) is a strongly stigmatised disease as it is framed within the context of injecting substance use. HCV provides the identity of 'dirty' or 'junky', with perceptions by others being beyond the control of the individual. People who experience problematic substance use are often viewed as being outside acceptable social behaviours, thus viewed as having tainted identities or second-class citizens. It is suggested that to recover from substance use, people should move towards social networks where substance use is not the norm and there is greater recovery support. The social identity model of recovery advocates that the mechanism to do this is by developing a new identity. It is unclear what catalysts provide this change in identity. This systematic review aims to describe actions, interventions and treatments that provide the opportunity for new identities and considers evidence that supports the hypothesis that curing HCV with direct acting antivirals may provide this opportunity.

Methods and analysis Methods are informed by the Preferred Reporting Items for Systematic reviews and Meta-Analysis statement. Seven electronic peer-reviewed and four grey literature sources were identified and preliminary searches have been conducted. The inclusion and exclusion criteria are broad to capture activities that result in a change in identity, recovery from substance use, quality of life, life satisfaction or the opportunity for the individual to reclaim their place in society (citizenship). Qualitative and quantitative literature are eligible. Papers will be assessed against standardised criteria and checked independently and in duplicate. A narrative synthesis of the findings will be reported, structured around intervention type, population context and outcomes.

Ethics and dissemination This systematic review will be based on studies that have already been conducted and therefore no ethical approvals are required. The resulting findings will be submitted to an international peerreviewed journal and disseminated at relevant research conferences.

PROSPERO registration number CRD42020209447.

\section{INTRODUCTION}

Problematic substance use has wide and far reaching health and social consequences for individuals and communities. ${ }^{12}$ Injecting
Strengths and limitations of this study

- To our knowledge it is the first systematic review on this topic.

- The study design includes a large grey literature search component to minimise publication bias and independent double-screening and coding to minimise selection bias and data errors.

- The study is restricted to English language evidence and has a Western-culture bias (although the latter is by design) which may limit application of findings.

- There is potential bias in the review design because of the research team's prior knowledge of hepatitis $\mathrm{C}$ virus treatment, recovery from substance use and citizenship literature which may have influenced the research questions.

drug use is strongly stigmatised within society; those who participate are regarded as acting outside of acceptable social norms and as a result are often viewed as having tainted identities and as second-class citizens. ${ }^{3}$

Health risks inherent with injecting drug use mean that people with a current or past history are the key risk group for hepatitis $\mathrm{C}$ virus (HCV) infection. ${ }^{4}$ As HCV is framed within this context, it is often perceived to be a shameful and stigmatising disease providing an identity of 'junkie', 'dirty', with perception by others being beyond the control of the individual. $^{35-9}$

The introduction of direct acting antiviral (DAA) drugs to treat HCV transformed care, providing highly effective ( $96 \%$ cure rate), simple and quick treatment regimes with few side effects. This is a vast improvement from care experienced in the interferon-era where treatment was arduous with only moderate cure rates. ${ }^{10-14}$ The paradigm shift to DAAs were heralded as a technology of hope, providing the stimulus to realise the WHO's target of eliminating HCV as a public health concern by 2030 through increased treatment 
uptake and reaching the most marginalised. ${ }^{5} 1011$ 15-17 However, while DAA treatment may be less onerous, significant barriers, such as stigma and competing priorities remain and further action is required to reach the key risk group to address the low number of people engaging with HCV testing and treatment. ${ }^{18-21}$

People who inject drugs are suggested to be looking for a cure beyond HCV as a reason to engage with treatment; the opportunity for an untainted identity, recovery from substance use and social redemption. ${ }^{22}$ Understanding and communicating how DAAs may provide these wider non-clinical benefits may present compelling evidence for this group to engage with treatment.

Identity transformation is a key component of recovery from substance use. ${ }^{23-28}$ Social identity provides a sense of who you are based on the group(s) of people with whom one associates with, which in turn shapes ones behaviours and influences health and well-being. ${ }^{262930}$ Social identity may provide support and positive influences on health behaviours, however, it may also influence negative lifestyle choices and create a barrier to recovery. ${ }^{24} 31$ The concept of a Social Identity Model of Recovery proposes that recovery from substance use relies on an intertwined shift in self-identity and social identity. The change in self-identity of the individual towards a 'recovery' identity shapes their social network to one where the use of drugs in not the social norm. ${ }^{23} 27$ This social network, away from substance use re-enforces the 'recovery' self-identity and also provides a recovery enabling environment, a key factor recognised in building recovery capital.

Recovery capital is a theoretical construct that describes the social, cultural, physical and human capital that can be brought to bear on the initiation and maintenance of recovery from dependent substance use. ${ }^{33}$ Recovery from substance use is defined as a process that encompasses other markers of progress towards overall health such as emotional well-being, and building and maintaining relationships. An integral part of recovery is reintegration into the community ${ }^{34}$; not just physical citizenship but also being part of society; being a citizen in everyday life. ${ }^{35}$

The catalyst that provides the change in self-identity that may propel an individual down a recovery pathway to reclaim their place in society remains unclear. The purpose of this review is to provide information about the types of interventions, treatments or actions that provide the opportunity for a change in identity and support a recovery journey. The review will also specifically consider if treatment for HCV with DAAs provides this catalyst. Developing this knowledge may provide compelling evidence to engage in testing and treatment at an individual and society level. The review may also identify new avenues for research for the delivery of the WHO targets for HCV elimination

\section{Objectives}

To our knowledge, no systematic reviews with a similar aim have been conducted previously. Guided by the research objectives numbered below, this systematic review aims: To

\begin{tabular}{|c|c|}
\hline Population & $\begin{array}{l}\text { People in recovery from dependent } \\
\text { substance use; illicit use and } \\
\text { prescription medicine misuse, } \\
\text { including alcohol and excluding } \\
\text { tobacco. }\end{array}$ \\
\hline $\begin{array}{l}\text { Intervention/ } \\
\text { Exposure }\end{array}$ & $\begin{array}{l}\text { Any action, treatment or intervention } \\
\text { for substance use or related health } \\
\text { issue. }\end{array}$ \\
\hline Comparator & Any or none. \\
\hline Outcomes & $\begin{array}{l}\text { The opportunity for a change in identity } \\
\text { and support for a recovery journey. }\end{array}$ \\
\hline Context & $\begin{array}{l}\text { From the UK and countries culturally } \\
\text { similar to the UK (USA, Canada, } \\
\text { Australia, New Zealand, Western } \\
\text { Europe). }\end{array}$ \\
\hline
\end{tabular}

review the existing literature on recovery from substance use and provide a comprehensive overview on the role a change in identity has in propelling an individual down a recovery pathway. To provide a commentary on the types of actions that may cause a change in identity and consider if treatment with DAAs for HCV provides this opportunity.

1. Determine interventions, treatments or actions related to substance use that result in a change in identity.

2. Describe the impact a change in identity has on a recovery journey, quality of life, life satisfaction and citizenship.

3. Consider evidence for change of identity as a result of treatment for HCV with DAAs.

\section{METHODS AND ANALYSIS}

The systematic review will include studies that meet the eligibility criteria and the objectives set in PI(E)COC (table 1) which outlines the criteria for the population, the intervention/exposures under review, comparator, outcomes of interest and context/setting of the study.

\section{Eligibility criteria}

The search will be conducted in the relevant databases and grey literature sources outlined in this protocol. The inclusion and exclusion criteria are broad to capture activities that result in a change in identity, recovery from substance use, quality of life or life satisfaction or the opportunity for the individual to reclaim their place in society (citizenship). Quantitative and qualitative studies will be included in this review.

\section{Population}

The population of interest are people with a reported current or past history of dependent substance use, defined by diagnosis or behavioural indicators, such as injecting drug use. Substance use may include illicit use or prescribed medication misuse (where use is for a purpose other than that prescribed and in a manner that has a negative impact on health and/or functioning) 
and dependent and harmful alcohol use. Tobacco use is excluded as it is outside the scope of this systematic review.

\section{Interventions and exposures}

Any action, treatment or intervention for substance use or related health issue, for example, HCV, HIV, hepatitis $\mathrm{B}$, bacterial infection, chronic pain and mental health problems will be included in this review. Health issues wider than HCV, but related to substance use have been included in this review as they are often intertwined and there may be transferable learning. Interventions, treatment or actions delivered via a community programme, citizenship programme, rehabilitation or treatment programme will be included in the systematic review. Community programmes and citizenship programmes are defined as structured programmes that enhance community and social opportunities for people who use drugs who experience social exclusion as a result of stigmatisation and marginalisation. Any treatment or intervention identified that does not report on recovery or a change in social or self-identity will be excluded. Papers without sufficient data to determine the inter-relatedness of the intervention, action or treatment and a change in identity or the connection between identity and recovery will also be excluded.

\section{Comparator}

Studies with no comparator or control are included in this systematic review as the purpose of the review is to identify treatments or interventions that result in the outcomes previously outlined. The purpose of the study is not to compare treatments or interventions.

\section{Outcome}

The studies must report outcomes of a recovery journey from substance use and a change in identity. Recovery can mean different things to different people. Recovery may be measured using quantitative or qualitative tools, described by participants or as a secondary measure. Recovery journeys may include, but are not restricted to, abstinence, reduced frequency of drug use, social functioning, employment status, community activity, citizenship (reclaiming place in society) and changes in self-identity towards a recovery identity, social identity change through a shift in social networks towards groups that provide support for recovery, recovery capital, selfconcept, quality of life and well-being. Studies that are related to recovery from substance use where there is no measure of a change in identity, change in quality of life, life satisfaction or citizenship (reclaiming place in society or undertaking activities thought of as normal expected activities of citizenship) are excluded.

\section{Context}

Primary qualitative and quantitative studies will be included which are published in the English Language since the year 1991, since this is the year that interferon became commercially available to treat HCV. The data must relate to people reporting a history of dependent substance use and be from countries culturally similar to the UK (USA, Canada, Australia, New Zealand and Western Europe) to allow appropriate comparisons to be made.

\section{Patient and public involvement}

Patients and public were not directly involved in the design of this study. As this is a protocol for a systematic review and no participant recruitment will take place.

\section{Information sources and search strategy}

The following electronic databases will be systematically searched using a mix of free text terms and indexed terms: MEDLINE, EMBASE, PsycINFO, ProQuest Public Health, ProQuest Sociological Abstracts, CINAHL and Web of Science (core collection from 2010 to 2020). The search strategy for the electronic databases is informed by the PI(E)COC components described above. It combines free text and database-specific index terms related to the Population (eg, terms for substance users/use, addiction and history of addiction); terms related to the Intervention or Exposure (eg, terms for intervention, treatment, therapy, rehabilitation, abstinence and recovery); and terms related to Outcomes (eg, terms for identity, citizenship, social norms, reinvent, stigma and social group). The strategy is limited to results published in 1991 to date, since this was the year that interferon was commercially available to treat HCV and limited to English language papers only. A grey literature search of conference material from the International Network on Hepatitis in Substance Users, Society for the Study of Addictions annual symposium, Lisbon Addictions conference and Improving Outcomes in the Treatment of Opioid Dependency conference from the year 2010 (where available online) will also be conducted to identify further studies and mitigate potential bias to the review from locating studies from peer-reviewed journals only. If additional studies are identified from the conference materials and full papers are not available, authors will be contacted for draft papers. Conference abstracts will be included if full or draft papers are not available. A copy of the search strategy is included in online supplemental appendix 1.

\section{DATA}

\section{Data selection and coding}

The results from the systematic search will be combined using EndNote (Clarivate) V.X9 and duplicate results removed. The remaining results will be evaluated by two authors (AR and SRD) who will perform an inclusion/exclusion criteria-based screening of the titles and abstracts. A minimum of $15 \%$ of preliminary screened articles will be screened together to ensure consistency in screening methodology and both authors will independently screen all results. EndNote V.X9 will be used to manage the screening process. Once the initial screening step is complete all full texts of the remaining results will be independently screened by two authors (AR and SRD). 
In cases of disagreements at each step, a third author (JFD) will be consulted to assist in reaching a decision.

A Preferred Reporting Items for Systematic reviews and Meta-Analysis (PRISMA) flow diagram will be included in the systematic review to describe the selection process. After the initial screening process the exclusion reason for full article screening will be recorded and reported using the PI(E)COC criteria set out in this protocol. If a result is excluded due to a number of criteria the reason for exclusion will follow the PI(E)COC order. For example, a result excluded due to participants and context will be recorded as exclusion due to participants.

\section{Data extraction}

Data will be extracted from the relevant studies. To allow for a narrative synthesis of qualitative and quantitative results, the discussion section of studies reporting quantitative results will be used for thematic data extraction. Key characteristics of study design, participant characteristics, results (including themes and quotes), context and author interpretations will be extracted into a spreadsheet and coded in NVivo (QSR international) V.12 independently and in duplicate by two authors (AR and SRD). Studies will be coded into pre-existing concepts, with new concepts were created when required. Any disagreements will be discussed by the two authors and if a decision cannot be reached, a third author (JFD) will be consulted.

\section{Risk of bias (quality) assessment}

The relevance of included selected studies will be assessed in duplicate by two authors (AR and SRD) using the study inclusion criteria (above), and the rigour and validity of included studies will be evaluated using the relevant Critical Appraisal Skills Programme (CASP) checklist for the study design. ${ }^{36}$ The CASP checklists will be used to systematically appraise the methodological quality of the studies. If a study methodology is considered to be inappropriate to address the aims of the research, it will be discussed by both authors (AR and SRD) to determine if it should be included in the review. In cases of disagreements, a third author (JFD) will be consulted to assist in reaching a decision.

\section{Synthesis and analysis}

The selected studies will be subject to thematic synthesis based on written information by the authors and data (eg, quotes/transcripts) documented. All studies selected for review will have the full texts read by both authors to identify and gain consensus of themes. Themes will be identified through an inductive approach through discussion between authors, Where there are disagreements, they will be discussed between both authors (AR and SRD) with a third author (JFD) consulted as arbitrator when required

If necessary data are available, studies will be grouped according to participant characteristics (such as gender, employment, education). As this is a qualitative synthesis, it is not possible to specify groups in advance. A narrative synthesis of the findings will be reported, structured around intervention type, population context, and outcomes.

This protocol adheres to the PRISMA-P 17 item checklist $^{3738}$ to ensure that the resulting systematic review is robust and transparent and any changes to the protocol will be reported and explained. A copy of this checklist is included in online supplemental appendix 1. The resulting systematic review will be checked against the PRISMA statement prior to submission for publication. ${ }^{39}$

\section{Potential implications}

This systematic review may provide information about the types of treatment or interventions that provide the catalyst for a change in identity which may propel an individual with (problematic/dependent) substance use down a recovery pathway. This review may provide evidence to suggest that treatment and cure of HCV using DAAs may provide the type of catalyst required to initiate this change, therefore may provide additional reason to engage with DAA treatments at an individual and societal level. Additionally, this systematic review may identify new avenues for further research.

\section{ETHICS AND DISSEMINATION}

This systematic review will be based on studies that have already been conducted and therefore no ethical approvals are required. This systematic review has been registered on PROSPERO and the resulting paper will be submitted to a peer-reviewed international journal. The resulting findings will also be disseminated at relevant research conferences. Any amendments to the protocol will be registered on PROSPERO.

\section{Review status}

At the time of submission, the systematic search has been completed and duplicate search results have been removed. The screening process of titles and abstracts has started and the planned review is expected to be complete by October 2021 with report submission in December 2021.

Twitter Sarah R Donaldson @donaldson_sarah and Andrew Radley @AndrRadl

Acknowledgements We thank Kathryn Angus (University of Stirling) for advice and comments on drafts of this protocol. She received no remuneration and was not funded for this work.

Contributors SRD wrote the protocol for the systematic review, performed the preliminary searches, will conduct data extraction, quality assessment and thematic analysis and will draft the systematic review paper. AR contributed to the design of the systematic review and will conduct data extraction, independent quality assessment and independent thematic analysis. JFD made substantial contributions to the design of the systematic review and, if required, will assist SRD and AR to resolve any discrepancies regarding study inclusion, data extraction and quality assessment. All authors contributed to revisions to the protocol manuscript and will contribute to the systematic review manuscript.

Funding This work is part of a project grant funded by Gilead Sciences Ltd (UK-HCV-2020-11-0056).

Competing interests SRD none declared, AR declares receipt of research grants from Abbvie, BMS, Pfizer and Gilead. Lecture fees and honorariums from Abbvie and Gilead. JFD declares receipt of research grants, lecture fees and honorariums 
from Abbvie, BMS, Gilead, MSD and Roche. The funder had no role in the design, execution, interpretation or writing of the study.

Patient consent for publication Not applicable.

Ethics approval This study does not involve human participants.

Provenance and peer review Not commissioned; externally peer reviewed.

Supplemental material This content has been supplied by the author(s). It has not been vetted by BMJ Publishing Group Limited (BMJ) and may not have been peer-reviewed. Any opinions or recommendations discussed are solely those of the author(s) and are not endorsed by BMJ. BMJ disclaims all liability and responsibility arising from any reliance placed on the content. Where the content includes any translated material, BMJ does not warrant the accuracy and reliability of the translations (including but not limited to local regulations, clinical guidelines, terminology, drug names and drug dosages), and is not responsible for any error and/or omissions arising from translation and adaptation or otherwise.

Open access This is an open access article distributed in accordance with the Creative Commons Attribution Non Commercial (CC BY-NC 4.0) license, which permits others to distribute, remix, adapt, build upon this work non-commercially, and license their derivative works on different terms, provided the original work is properly cited, appropriate credit is given, any changes made indicated, and the use is non-commercial. See: http://creativecommons.org/licenses/by-nc/4.0/.

\section{ORCID iDs}

Sarah R Donaldson http://orcid.org/0000-0003-2816-3293

Andrew Radley http://orcid.org/0000-0003-4772-2388

\section{REFERENCES}

1 Clinical Guidelines on Drug Misuse and Dependence Update 2017 Independent Expert Working Group. Drug misuse and dependence: UK guidelines on clinical management. 25460. London: Global and Public Health / Population Health / Healthy Behaviours, 2017.

2 The Scottish Government. Rights, Respect and Recovery. Scotland's strategy to improve health by preventing and reducing alcohol and drug use, harm and related deaths. Edinburgh, 2018.

3 Treloar C, Rance J, Backmund M. Understanding barriers to hepatitis $\mathrm{C}$ virus care and stigmatization from a social perspective. Clin Infect Dis 2013;57 Suppl 2:S51-5.

4 European Monitoring Centre for Drugs and Drug Addiction (EMCDDA). Hepatitis C among drug users in Europe: epidemiology, treatment and prevention. Luxembourg: publications office of the European Union, 2016.

5 Rhodes T, Harris M, Martin A. Negotiating access to medical treatment and the making of patient citizenship: the case of hepatitis C treatment. Sociol Health IIIn 2013;35:1023-44.

6 Rhodes T, Treloar C. The social production of hepatitis C risk among injecting drug users: a qualitative synthesis. Addiction 2008;103:1593-603.

7 Harris M. Injecting, infection, illness: Abjection and hepatitis C stigma. Body Soc 2009;15:33-51.

8 Dowsett LE, Coward S, Lorenzetti DL, et al. Living with hepatitis $C$ virus: a systematic review and narrative synthesis of qualitative literature. Can J Gastroenterol Hepatol 2017;2017:1-11.

9 Treloar C, Rhodes T. The lived experience of hepatitis $C$ and its treatment among injecting drug users: qualitative synthesis. Qual Health Res 2009;19:1321-34.

10 World Health organization. Combating hepatitis $B$ and $C$ to reach elimination by 2030: advocacy brief, 2016. Available: https://apps. who.int/iris/handle/10665/206453

11 Harris M, Rhodes T. Caring and curing: considering the effects of hepatitis $C$ pharmaceuticalisation in relation to non-clinical treatment outcomes. Int J Drug Policy 2018;60:24-32.

12 Clark JA, Gifford AL. Resolute efforts to cure hepatitis C: understanding patients' reasons for completing antiviral treatment. Health 2015;19:473-89.

13 Bonaccorso S, Marino V, Biondi M, et al. Depression induced by treatment with interferon-alpha in patients affected by hepatitis $\mathrm{C}$ virus. J Affect Disord 2002;72:237-41.
14 Körner H. Negotiating treatment for hepatitis C: interpersonal alignment in the clinical encounter. Health 2010;14:272-91.

15 World Health Organization. Global hepatitis report 2017, 2017. Available: https://www.who.int/publications/i/item/global-hepatitisreport-2017

16 Bruggmann P, Grebely J, Prevention GJ. Prevention, treatment and care of hepatitis $C$ virus infection among people who inject drugs. Int J Drug Policy 2015;26 Suppl 1:S22-6.

17 Lazarus JV, Wiktor S, Colombo M, et al. Micro-elimination - A path to global elimination of hepatitis C. J Hepatol 2017;67:665-6.

18 Razavi H, Sanchez Gonzalez Y, Yuen C, et al. Global timing of hepatitis $\mathrm{C}$ virus elimination in high-income countries. Liver Int 2020;40:522-9.

19 Hickman M, Martin NK, Giraudon I. Hepatitis C among drug users in Europe: epidemiology treatment and preventio, 2016. Available: https://www.emcdda.europa.eu/system/files/publications/2953/ TDXD16002ENN_final_web.pdf

20 Davis M, Rhodes T. Beyond prevention? injecting drug user narratives about hepatitis C. International Journal of Drug Policy 2004;15:123-31.

21 Bryant J, Rance J, Hull P, et al. Making sense of 'side effects': Counterpublic health in the era of direct-acting antivirals. Int J Drug Policy 2019;72:77-83.

22 Madden A, Hopwood M, Neale J, et al. Beyond cure: patient reported outcomes of hepatitis $C$ treatment among people who inject drugs in Australia. Harm Reduct $J$ 2018;15:42.

23 Dingle GA, Cruwys T, Frings D. Social identities as pathways into and out of addiction. Front Psychol 2015;6:12.

24 Frings D, Albery IP. The social identity model of cessation maintenance: formulation and initial evidence. Addict Behav 2015;44:35-42.

25 Praharso NF, Tear MJ, Cruwys T. Stressful life transitions and wellbeing: a comparison of the stress buffering hypothesis and the social identity model of identity change. Psychiatry Res 2017;247:265-75.

26 Mawson E, Best D, Beckwith M, et al. Social identity, social networks and recovery capital in emerging adulthood: a pilot study. Subst Abuse Treat Prev Policy 2015;10:45.

27 Best D, Beckwith M, Haslam C, et al. Overcoming alcohol and other drug addiction as a process of social identity transition: the social identity model of recovery (SIMOR). Addict Res Theory 2016:24:111-23.

28 McIntosh J, McKeganey N. Addicts' narratives of recovery from drug use: constructing a non-addict identity. Soc Sci Med 2000;50:1501-10.

29 West R. European monitoring centre of drugs and drug addiction (EMCDDA), insights. models of addiction. EMCDDA. Luxembourg: Publications Office of the European Union, Lisbon, Portugal, 2013.

30 Giovazolias T, Themeli O. Social learning conceptualization for substance abuse: implications for therapeutic interventions. Eur. J. Couns. Psych. 2014;3:69-88.

31 Jetten J, Haslam C, Alexander SH. The social cure: identity health and well-being. Psychology press, 2012.

32 Bathish R, Best D, Savic M, et al. "Is it me or should my friends take the credit?" The role of social networks and social identity in recovery from addiction. J App/ Soc Psychol 2017;47:35-46.

33 Cloud W, Granfield R. Conceptualizing recovery capital: expansion of a theoretical construct. Subst Use Misuse 2008;43:1971-86.

34 Best D, Colman C. Let's celebrate recovery. Inclusive Cities working together to support social cohesion. Addict Res Theory 2019;27:55-64.

35 Vervliet M, Reynaert D, Verelst A, et al. "If You Can't Follow, You're Out." The Perspectives of People with Mental Health Problems on Citizenship. Appl Res Qual Life 2019;14:891-908.

36 Critical Appraisal Skills Programme. Casp checklists, 2020. Available: https://casp-uk.net/casp-tools-checklists/

37 Shamseer L, Moher D, Clarke M, et al. Preferred reporting items for systematic review and meta-analysis protocols (PRISMA-P) 2015: elaboration and explanation. BMJ 2015;349:97647.

38 Moher D, Shamseer L, Clarke M, et al. Preferred reporting items for systematic review and meta-analysis protocols (PRISMA-P) 2015 statement. Syst Rev 2015;4:1.

39 Moher D, Liberati A, Tetzlaff J, et al. Preferred reporting items for systematic reviews and meta-analyses: the PRISMA statement. PLOS Med 2009;6:e1000097. 